PROCEEDINGS OF THE

AMERICAN MATHEMATICAL SOCIETY

Volume 131 , Number 12, Pages 3827-3832

S 0002-9939(03)07020-5

Article electronically published on April 24, 2003

\title{
ASYMPTOTIC LIMIT FOR CONDENSATE SOLUTIONS IN THE ABELIAN CHERN-SIMONS HIGGS MODEL II
}

\author{
JONGMIN HAN
}

(Communicated by David S. Tartakoff)

\begin{abstract}
In this paper we show that the maximal condensate solutions $\left(\phi^{\epsilon}, A^{\epsilon}\right)$ in the Abelian Chern-Simons Higgs model converge to $\left(\phi_{*}, A_{*}\right)$ in higher norms, where $\phi_{*}$ is a harmonic map.
\end{abstract}

\section{INTRODUCTION}

In this paper we continue our study discussed in [4] on asymptotic behaviors of the maximal solutions of the following equations on $\Omega$ :

$$
\begin{aligned}
D_{1} \phi+i D_{2} \phi & =0, \\
F_{A}+\frac{2}{\epsilon^{2}}|\phi|^{2}\left(|\phi|^{2}-1\right) & =0 .
\end{aligned}
$$

Here $\epsilon>0, \phi: \Omega \rightarrow \mathbb{C}$ is the complex Higgs field, $A: \Omega \rightarrow \mathbb{R}^{2}$ is the coupled gauge potential, $D_{A} \phi=\nabla \phi-i A \phi$ is the covariant derivative, and $F_{A}=\operatorname{curl} A$ is the magnetic field. The domain $\Omega$ is a basic lattice cell in $\mathbb{R}^{2}$ generated by two independent vectors $\mathbf{a}^{1}$ and $\mathbf{a}^{2}$, namely,

$$
\Omega=\left\{x \in \mathbb{R}^{2} \mid x=s_{1} \mathbf{a}^{1}+s_{2} \mathbf{a}^{2}, 0<s_{1}, s_{2}<1\right\} .
$$

The equations (1.1) and (1.2) arise from the self-dual Abelian Chern-Simons Higgs model proposed by Hong-Kim-Pac [5] and Jackiw-Weinberg [6]. It is easy to see that (1.1) and (1.2) are invariant under the gauge transformation

$$
(\phi, A) \rightarrow\left(e^{i \chi} \phi, A+\nabla \chi\right),
$$

where $\chi: \Omega \rightarrow \mathbb{R}$. In view of gauge invariance we impose the following 't Hooft boundary condition on $\Omega$ :

$$
\begin{aligned}
\exp \left(i \xi_{j}\left(x+\mathbf{a}^{j}\right)\right) \phi\left(x+\mathbf{a}^{j}\right) & =\exp \left(i \xi_{j}(x)\right) \phi(x), \\
\left(A_{k}+\partial_{k} \xi_{j}\right)\left(x+\mathbf{a}^{j}\right) & =\left(A_{k}+\partial_{k} \xi_{j}\right)(x), \quad k=1,2, \\
x \in \Gamma^{1} \cup \Gamma^{2}-\Gamma^{j}, & j=1,2,
\end{aligned}
$$

where $\xi_{1}$ and $\xi_{2}$ are real-valued smooth functions defined in a neighborhood of $\Gamma^{2} \cup\left\{\mathbf{a}^{1}+\Gamma^{2}\right\}, \Gamma^{1} \cup\left\{\mathbf{a}^{2}+\Gamma^{1}\right\}$, respectively. Here

$$
\Gamma^{j}=\left\{x \in \mathbf{R}^{2} \mid x=s \mathbf{a}^{j}, 0<s<1\right\}, \quad j=1,2 .
$$

Received by the editors July 24, 2002.

2000 Mathematics Subject Classification. Primary 35B40, 81T13.

Key words and phrases. Chern-Simons-Higgs model, self-duality equations, maximal solutions.

This research was supported by the research fund of Hankuk University of Foreign Studies, 2002. 
The solutions of (1.1) and (1.2) on $\Omega$ under the above boundary conditions are called condensate solutions.

Using the classical Jaffe-Taubes arguments [7], one can show that the equation (1.1) implies that $\phi$ is holomorphic up to a nonvanishing multiple factor and has exactly $N$ zeros allowing multiplicities. Thus in light of gauge invariance we may assume that $\phi$ takes the form

$$
\phi(x)=\exp \left(\frac{1}{2} u(x)+i \sum_{j=1}^{k} n_{j} \arg \left(x-p_{j}\right)\right),
$$

where the points $p_{1}, \cdots, p_{k}$, called the vortex points, are the distinct zeros of $\phi$ with multiplicities $n_{1}, \cdots, n_{k}$, respectively. Then the equation (1.2) can be reduced to

$$
\begin{aligned}
& \Delta u=\frac{4}{\epsilon^{2}} e^{u}\left(e^{u}-1\right)+4 \pi \sum_{j=1}^{k} n_{j} \delta_{p_{j}} \quad \text { on } \Omega, \\
& u: \text { doubly periodic. }
\end{aligned}
$$

Here $\delta_{p}$ denotes the Dirac measure concentrated on the point $p$. Conversely, once we find a solution $u$ of (1.5), we may recover $A$ from (1.1) by the formula

$$
A_{1}+i A_{2}=-2 i \bar{\partial} \ln \phi
$$

where $\bar{\partial}=\left(\partial_{1}+i \partial_{2}\right) / 2$.

The first result about the existence of solutions of (1.1) and (1.2) was given by Caffarelli-Yang [2]. They showed that there is a critical value $\epsilon_{c}<\sqrt{|\Omega| / 4 \pi N}$ so that for $0<\epsilon<\epsilon_{c}$ the equations (1.1) $\epsilon_{\epsilon}$ and $(1.2)_{\epsilon}$ with the boundary condition (1.3) admits a maximal solution $\left(\phi^{\epsilon}, A^{\epsilon}\right)$ of the form (1.4) and (1.6). The solution $\left(\phi^{\epsilon}, A^{\epsilon}\right)$ is maximal in the sense that $\left|\phi^{\epsilon}\right|$ has the largest possible value among all the solutions to $(1.1)_{\epsilon}$ and $(1.2)_{\epsilon}$ with the same zeros and multiplicities. In 8 it was proved by Tarantello that there exists another solution, and some asymptotic behaviors of solutions as $\epsilon \rightarrow 0$ were studied. See also [3] and references therein for recent progress for related topics.

In 4 we showed that

Theorem 1.1. Let $\left(\phi^{\epsilon}, A^{\epsilon}\right)$ be the maximal solution of (1.1) and (1.2) corresponding to $\epsilon$. Let $\Omega^{\prime}=\Omega \backslash\left\{p_{1}, \cdots, p_{k}\right\}$. Then for each $\alpha \in(0,1)$, we have

$$
\left(\phi^{\epsilon}, A^{\epsilon}\right) \rightarrow\left(\phi_{*}, A_{*}\right) \quad \text { in } \quad C_{l o c}^{1, \alpha}\left(\Omega^{\prime}, \mathbb{C}\right) \times C_{l o c}^{0, \alpha}\left(\Omega^{\prime}, \mathbb{R}^{2}\right)
$$

as $\epsilon \rightarrow 0$, where $\left(\phi_{*}, A_{*}\right)$ belongs to $W_{\text {loc }}^{2, p}\left(\Omega^{\prime}, \mathbb{C}\right) \times W_{l o c}^{1, p}\left(\Omega^{\prime}, \mathbb{R}^{2}\right)$ for all $p>1$ and satisfies

$$
\begin{aligned}
\Delta \phi_{*}+\phi_{*}\left|\nabla \phi_{*}\right|^{2} & =0, \\
\left|\phi_{*}\right| & =1, \\
\operatorname{deg}\left(\phi_{*}, p_{j}\right) & =n_{j}, \\
A_{*} & =-i \overline{\phi_{*}} \nabla \phi_{*}
\end{aligned}
$$

on $\Omega^{\prime}$. In fact,

$$
\phi_{*}(z)=\prod_{j=1}^{k} \frac{\left(z-p_{j}\right)^{n_{j}}}{\left|z-p_{j}\right|^{n_{j}}}
$$

on $\Omega^{\prime}$. 
The purpose of the present paper is to obtain the speed of the convergence of $\left(\phi^{\epsilon}, A^{\epsilon}\right)$ to $\left(\phi_{*}, A_{*}\right)$ in higher norms. In fact, we establish

Theorem 1.2. Let $\left(\phi^{\epsilon}, A^{\epsilon}\right)$ be the maximal solution of (1.1) and (1.2) corresponding to $\epsilon$. Let $\Omega^{\prime}=\Omega \backslash\left\{p_{1}, \cdots, p_{k}\right\}$. Then for each positive integer $s$, we have

$$
\left(\phi^{\epsilon}, A^{\epsilon}\right) \rightarrow\left(\phi_{*}, A_{*}\right) \quad \text { in } \quad C_{l o c}^{s}\left(\Omega^{\prime}, \mathbb{C}\right) \times C_{l o c}^{s-1}\left(\Omega^{\prime}, \mathbb{R}^{2}\right) .
$$

Furthermore for each $K \subset \subset \Omega^{\prime}$, as $\epsilon \rightarrow 0$,

$$
\left\|\phi^{\epsilon}-\phi_{*}\right\|_{C^{s}(K)} \leq C_{K, s} \epsilon^{2}
$$

and

$$
\left\|A^{\epsilon}-A_{*}\right\|_{C^{s-1}(K)} \leq C_{K, s} \epsilon^{2} .
$$

In the next section we give a proof of Theorem 1.2

\section{Proof of Theorem 1.2}

We notice that the equation (1.5) can be regarded as an exponential nonlinearity version of the following Ginzburg-Landau equations:

$$
-\Delta v=\frac{1}{\epsilon^{2}} v\left(1-|v|^{2}\right) \quad \text { in } \omega,
$$

where $v: \omega \subset \mathbb{R}^{2} \rightarrow \mathbb{C}$. The main strategy of the proof of Theorem 1.2 is to apply the same arguments used in [1] to describe the asymptotic behavior of solutions of (2.1).

Let us denote

$$
\Theta(x)=2 \sum_{j=1}^{k} n_{j} \arg \left(x-p_{j}\right) .
$$

Then it comes from (1.4), (1.6), (1.7), and (1.8) that

$$
\begin{aligned}
\phi^{\epsilon} & =\exp \left(u_{\epsilon} / 2+i \Theta / 2\right), \\
2 A^{\epsilon} & =\operatorname{curl} u^{\epsilon}+\nabla \Theta, \\
\phi_{*} & =\exp (i \Theta / 2), \\
2 A_{*} & =\nabla \Theta
\end{aligned}
$$

where $\operatorname{curl} u=\left(\partial_{2} u,-\partial_{1} u\right)$.

In order to prove Theorem 1.2, we need some lemmas.

Lemma 2.1 (1]). Let $U$ be a bounded domain in $\mathbb{R}^{n}$. Suppose that

$$
\Delta v=f \quad \text { in } U \text {. }
$$

Then for each $K \subset \subset U$,

$$
\|\nabla v\|_{L^{\infty}(K)}^{2} \leq C_{K}\|v\|_{L^{\infty}(U)}\left(\|f\|_{L^{\infty}(U)}+\|v\|_{L^{\infty}(U)}\right) .
$$

Lemma 2.2 (3]). For every compact subset $K$ of $\Omega \backslash\left\{p_{1}, \cdots, p_{k}\right\}$,

$$
0 \leq 1-\left|\phi^{\epsilon}(x)\right|^{2} \leq C_{K} \epsilon^{2}, \quad \forall x \in K,
$$

as $\epsilon \rightarrow 0$.

Lemma 2.3. For each $K \subset \subset \Omega^{\prime}$ and nonnegative integer $s$, we have

$$
\left\|1-e^{u_{\epsilon}}\right\|_{C^{s}(K)} \leq C_{K, s} \epsilon^{2}
$$

as $\epsilon \rightarrow 0$. 
Proof. The proof is given by induction. The case $s=0$ follows from (2.2) and we suppose that (2.3) holds up to $s \geq 0$.

Let us choose $x_{0} \in \Omega^{\prime}$ and $R<\inf \left\{\left|x_{0}-p_{j}\right| / 5: j=1, \cdots, k\right\}$. Set

$$
w_{\epsilon}=\frac{1-e^{u_{\epsilon}}}{\epsilon^{2}} \text {. }
$$

Then it comes from (1.5) that

$$
\Delta u_{\epsilon}=4 \epsilon^{2} w_{\epsilon}^{2}-4 w_{\epsilon} \quad \text { on } \quad B_{5 R}\left(x_{0}\right),
$$

where $B_{5 R}\left(x_{0}\right)$ is a ball of radius $5 R$ centered at $x_{0}$. Since $\left\|w_{\epsilon}\right\|_{C^{s}\left(B_{5 R}\right)} \leq C$ by induction assumption, it follows from the elliptic estimates that $\left\|u_{\epsilon}\right\|_{W^{s+2, p}\left(B_{4 R}\right)} \leq$ $C$ for all $p>1$. In particular,

$$
\left\|u_{\epsilon}\right\|_{C^{s+1}\left(B_{4 R}\right)} \leq C
$$

A short computation yields

$$
\Delta w_{\epsilon}=\frac{4}{\epsilon^{2}} e^{2 u_{\epsilon}} w_{\epsilon}-\frac{1}{\epsilon^{2}} e^{u_{\epsilon}}\left|\nabla u_{\epsilon}\right|^{2} \equiv f_{\epsilon} \quad \text { on } \quad B_{4 R} .
$$

Then by Lemma 2.1

$$
\begin{aligned}
& \left\|\partial^{s+1} w_{\epsilon}\right\|_{L^{\infty}\left(B_{3 R}\right)}^{2} \\
\leq & C\left\|\partial^{s} w_{\epsilon}\right\|_{L^{\infty}\left(B_{4 R}\right)}\left(\left\|\partial^{s} w_{\epsilon}\right\|_{L^{\infty}\left(B_{4 R}\right)}+\left\|\partial^{s} f_{\epsilon}\right\|_{L^{\infty}\left(B_{4 R}\right)}\right) \\
\leq & C\left(1+\frac{1}{\epsilon^{2}}\left\|w_{\epsilon}\right\|_{C^{s}\left(B_{4 R}\right)} \cdot\left\|u_{\epsilon}\right\|_{C^{s+1}\left(B_{4 R}\right)}\right) \\
\leq & C \epsilon^{-2} .
\end{aligned}
$$

Hence we have

$$
\left\|\partial^{s+1} w_{\epsilon}\right\|_{L^{\infty}\left(B_{3 R}\right)} \leq C \epsilon^{-1} .
$$

Differentiating (2.4) $(s+1)$ times and applying Lemma 2.1, we deduce from (2.5) and (2.7) that

$$
\begin{aligned}
& \left\|\partial^{s+2} u_{\epsilon}\right\|_{L^{\infty}\left(B_{2 R}\right)}^{2} \\
\leq & C\left\|\partial^{s+1} u_{\epsilon}\right\|_{L^{\infty}\left(B_{3 R}\right)}\left(\left\|\partial^{s+1} u_{\epsilon}\right\|_{L^{\infty}\left(B_{3 R}\right)}+\left\|\Delta \partial^{s+1} u_{\epsilon}\right\|_{L^{\infty}\left(B_{3 R}\right)}\right) \\
\leq & C\left(1+\left\|\partial^{s+1} w_{\epsilon}\right\|_{L^{\infty}\left(B_{3 R}\right)}\right) \\
\leq & C \epsilon^{-1} .
\end{aligned}
$$

Thus

$$
\left\|\partial^{s+2} u_{\epsilon}\right\|_{L^{\infty}\left(B_{2 R}\right)} \leq C \epsilon^{-1 / 2} .
$$

Next differentiating (2.6) $(s+1)$ times and applying Lemma 2.1 again, we find by (2.7) and (2.8) that

$$
\begin{aligned}
& \left\|\partial^{s+2} w_{\epsilon}\right\|_{L^{\infty}\left(B_{R}\right)}^{2} \\
\leq & C\left\|\partial^{s+1} w_{\epsilon}\right\|_{L^{\infty}\left(B_{2 R}\right)}\left(\left\|\partial^{s+1} w_{\epsilon}\right\|_{L^{\infty}\left(B_{2 R}\right)}+\left\|\partial^{s+1} f_{\epsilon}\right\|_{L^{\infty}\left(B_{2 R}\right)}\right) \\
\leq & \frac{C}{\epsilon^{2}}\left\|\partial^{s+1} w_{\epsilon}\right\|_{L^{\infty}\left(B_{2 R}\right)}\left(\left\|\partial^{s+1} w_{\epsilon}\right\|_{L^{\infty}\left(B_{2 R}\right)}+\left\|\partial^{s+2} u_{\epsilon}\right\|_{L^{\infty}\left(B_{2 R}\right)}\right) \\
\leq & C \epsilon^{-4} .
\end{aligned}
$$


Therefore

$$
\left\|\partial^{s+2} w_{\epsilon}\right\|_{L^{\infty}\left(B_{R}\right)} \leq C \epsilon^{-2} .
$$

Now let us rewrite (2.6) as

$$
-\epsilon^{2} \Delta w_{\epsilon}+4 w_{\epsilon}=8 \epsilon^{2} w_{\epsilon}^{2}-4 \epsilon^{4} w_{\epsilon}^{3}-\epsilon^{2} \nabla u_{\epsilon} \cdot \nabla w_{\epsilon} \equiv h_{\epsilon} \quad \text { on } \quad B_{R} .
$$

Then

$$
-\epsilon^{2} \Delta \partial^{s+1} w_{\epsilon}+4 \partial^{s+1} w_{\epsilon}=\partial^{s+1} h_{\epsilon} \quad \text { on } \quad B_{R} .
$$

It is seen from (2.5) $,(2.7),(2.8)$, and (2.9) that

$$
\left\|\partial^{s+1} h_{\epsilon}\right\|_{L^{\infty}\left(B_{R}\right)} \leq C .
$$

Set

$$
v=\partial^{s+1} w_{\epsilon}-\frac{\left\|\partial^{s+1} h_{\epsilon}\right\|_{L^{\infty}\left(B_{R}\right)}}{4} .
$$

From (2.7) we are led to

$$
\begin{array}{rlrl}
-\epsilon^{2} \Delta v+4 v & \leq 0 & \text { in } \quad B_{R}, \\
v & \leq \frac{C}{\epsilon}-\frac{\left\|\partial^{s+1} h_{\epsilon}\right\|_{L^{\infty}\left(B_{R}\right)}}{4} & \text { on } & \partial B_{R} .
\end{array}
$$

On the other hand it is easy to check that for $\epsilon<R$, the function

$$
V(r)=\frac{C}{\epsilon} \exp \left(\frac{1}{2 \epsilon R}\left(r^{2}-R^{2}\right)\right), \quad r=\left|x-x_{0}\right|
$$

is a supersolution of (2.11). This implies that

$$
\partial^{s+1} w_{\epsilon}(r) \leq \frac{\left\|\partial^{s+1} h_{\epsilon}\right\|_{L^{\infty}\left(B_{R}\right)}}{4}+\frac{C}{\epsilon} \exp \left(-\frac{3 R}{8 \epsilon}\right) \quad \text { on } B_{R / 2} .
$$

Similarly,

$$
\partial^{s+1} w_{\epsilon}(r) \geq-\frac{\left\|\partial^{s+1} h_{\epsilon}\right\|_{L^{\infty}\left(B_{R}\right)}}{4}-\frac{C}{\epsilon} \exp \left(-\frac{3 R}{8 \epsilon}\right) \quad \text { on } B_{R / 2} .
$$

As a consequence we conclude that

$$
\left\|\partial^{s+1} w_{\epsilon}\right\|_{L^{\infty}\left(B_{R / 2}\right)} \leq C,
$$

and the proof is completed.

Corollary 2.4. For each $K \subset \subset \Omega^{\prime}$ and nonnegative integer $s$, we have

$$
\left\|\nabla u_{\epsilon}\right\|_{C^{s}(K)} \leq C_{K, s} \epsilon^{2}
$$

as $\epsilon \rightarrow 0$.

Proof. By (2.2) we may assume that $e^{u_{\epsilon}} \geq 1 / 2$ on $K$ as $\epsilon \rightarrow 0$. Then (2.12) immediately follows from (2.3).

We are now in a position to prove Theorem 1.2. Given $K \subset \subset \Omega^{\prime}$, we may suppose by (2.2) that $e^{u_{\epsilon}} \geq 1 / 2$ on $K$. Then we observe from (2.3) that

$$
\left\|1-e^{u_{\epsilon} / 2}\right\|_{C^{s}(K)} \leq C_{K, s} \epsilon^{2} .
$$

In the sequel (1.9) and (1.10) is verified from (2.12) and (2.13) by the formula

$$
\begin{aligned}
& \phi_{*}-\phi^{\epsilon}=\left(1-e^{u_{\epsilon} / 2}\right) e^{i \Theta / 2}, \\
& A_{*}-A^{\epsilon}=\frac{1}{2} \operatorname{curl} u_{\epsilon} .
\end{aligned}
$$




\section{REFERENCES}

1. F. Bethuel, H. Brezis and F. Hélein, Asymptotics for the minimization of a Ginzburg-Landau functional, Calc. Var. P.D.E. 1 (1993), pp. 123-148. MR 94m:35083

2. L. A. Caffarelli and Y. Yang, Vortex condensation in Chern-Simons-Higgs model: an existence theorem, Comm. Math. Phys. 168 (1995), pp. 321-336. MR 96b:81076

3. J. Han, Asymptotics for the vortex condensate solutions in Chern-Simons-Higgs theory, Asymptotic Anal. 28 (2001), 31-48. MR 2002m:58027

4. J. Han, Asymptotic limit for condensate solutions in the Abelian Chern-Simons Higgs model, Proc. Amer. Math. Soc. 131 (2003), 1839-1845.

5. J. Hong, Y. Kim, and P. Y. Pac, Multivortex Solutions of the Abelian Chern-Simons-Higgs Theory, Phys. Rev. Lett. 64 (1990), pp. 2230-2233. MR 91a:81115

6. R. Jackiw and E. J. Weinberg, Self-dual Chen-Simons Vortices, Phys. Rev. Lett. 64 (1990), 2234-2237. MR 91a:81117

7. A. Jaffe and C.H. Taubes, Vortices and Monopoles, Birkhäuser, Boston, 1980. MR 82m:81051

8. G. Tarantello, Multiple condensate solutions for the Chern-Simons-Higgs theory, J. Math. Phys. 37 (1996), pp. 3769-3796. MR 97f:58045

Department of Mathematics, Hankuk University of Foreign Studies, 89 Wangsan-ri Mohyun, Yongin, KyoungGi-do 449-791, Korea

E-mail address: jmhan@hufs.ac.kr 